Cahiers $d u$ MONDE RUSSE

\section{Cahiers du monde russe}

Russie - Empire russe - Union soviétique et États indépendants

$59 / 4 \mid 2018$

Varia

\title{
Karl Schlögel, Das sowjetische Jahrhundert
}

Archäologie einer untergangenen Welt

\section{Gábor T. Rittersporn}

\section{(2) OpenEdition}

12 Journals

Édition électronique

URL : https://journals.openedition.org/monderusse/10732

DOI : $10.4000 /$ monderusse. 10732

ISSN : $1777-5388$

Éditeur

Éditions de l'EHESS

\section{Édition imprimée}

Date de publication : 1 octobre 2018

Pagination : 648-651

ISBN : 978-2-7132-2747-9

ISSN : 1252-6576

\section{Référence électronique}

Gábor T. Rittersporn, « Karl Schlögel, Das sowjetische Jahrhundert », Cahiers du monde russe [En ligne], 59/4 | 2018, mis en ligne le 01 octobre 2018, consulté le 08 janvier 2022. URL : http://

journals.openedition.org/monderusse/10732 ; DOI : https://doi.org/10.4000/monderusse.10732

(c) École des hautes études en sciences sociales 
URSS

\section{Karl SCHLÖGEL}

\section{Das sowjetische Jahrhundert}

\section{Archäologie einer untergangenen Welt}

Munich : C. H. Beck, 2017, 912 p.

Comment décrire un monde disparu ? Comment saisir ce qui l'a constitué, ce qui l'a défait, ce qui en est définitivement perdu, ce qui en survit, ce qu'il a signifié pour les gens qui l'ont peuplé et pour leurs contemporains qui ne l'habitaient pas ? Comment saisir ce qu'il signifie pour ceux qui habitent aujourd'hui ce qui en reste et pour ceux qui les regardent de loin?

On pourrait croire que les historiens sont là pour répondre à ces questions. Mais sont-ils à même de dépasser les sujets plus ou moins circonscrits de leurs travaux pour rendre compte de tout un univers ? Osent-ils s'exposer à l'accusation de s'écarter notablement des conventions méthodologiques de leur métier pour pratiquer un genre inclassable dans les catégories consacrées de la profession? Peuvent-ils s'autoriser à fonder leur narration sur un luxe de publications connues, peu connues, voire inconnues ou ignorées de la plupart de leurs collègues au lieu de s'appliquer à déchiffrer des sources débusquées dans les sacro-saintes archives? Se risquent-ils à affronter le jugement de la corporation qui peut reconnaître avec condescendance quelques mérites à leur démarche et en même temps condamner l'abandon de l'exposé systématique des résultats de recherches savantes au profit d'un récit érudit qui obéirait à la logique de la création littéraire ?

Karl Schlögel brave les règles et les interdits, écrits et sous-entendus, de la recherche et de la narration historiques. Il s'aventure vers des horizons aussi larges que l'ensemble des expériences vécues, telles qu'elles se déploient dans le temps, d'une société fragmentée, divisée et répartie dans une multitude déconcertante de champs, strictement séparés, tangentiels ou imbriqués. Il explore l'évolution d'une société aux dimensions démographiques et culturelles hors normes, il explore des lieux et des milieux de l'existence ordinaire et exceptionnelle de cette société et les objets emblématiques qui les meublent y compris les corps humains réifiés. Il présente une chronique qui s'empare du lecteur dès la première page, une histoire au sens original du terme dont le courant est si puissant que le lecteur ne s'en arrache 
pas facilement. Autant dire que ce livre de huit cent quarante-cinq pages qui parle de choses on ne peut plus complexes se lit aussi simplement qu'un roman qu'on regrette de quitter après l'épilogue.

Pourtant, cette histoire n'est pas structurée par des événements proprement dits. Certes, elle est construite à partir d'éléments qui sont inséparables d'activités humaines et parfois d'actions ponctuelles. Cependant, excepté le récit envoûtant des jours mouvementés pendant lesquels les bolcheviks se sont emparés des infrastructures du pouvoir d'État que Schlögel décrit en juxtaposant les témoignages de John Reed et de Nikolaj Suhanov, il n'y a guère de chapitres dans l'ouvrage où l'action est au centre de la narration. Schlögel fait visiter des endroits typiques, des espaces parlants, des objets topiques, il explore la signification cachée et la charge émotionnelle de situations et de circonstances révélatrices, il décrit des attitudes et des réflexes caractéristiques. Il est à la recherche du sens ou des sens qu'on peut leur donner en tant que manifestations matérielles, comportementales et spirituelles de l'histoire d'une société et d'une culture, il étudie les relations humaines et les arrangements institutionnels qu'elles suscitent et qui les façonnent. Il pratique une phénoménologie des choses soviétiques au lieu de raconter les travaux et les jours de l'URSS.

Schlögel part de l'observation d'un endroit plutôt inhabituel pour l'historien qui se respecte. Il s'agit de la baraholka d'Izmajlovo, du marché aux puces moscovite et de ses memorabilia sovietiche, de l'inventaire à la Prévert d'objets que des brocanteurs vendent comme souvenirs du pays défunt des soviets. Autant de trouvailles précieuses pour les fouilles archéologiques qu'il entreprend. On les retrouve par exemple quand Schlögel explique l'importance des ordres et des médailles, la signification du mobilier et des bibelots ou les mérites du téléphone, appareil drôlement vieillot sur les étalages mais pendant longtemps symbole de pouvoir et de prestige dans une société où il était rare, convoité et attribut indispensable des cabinets des cadres de l'État-Parti, des demeures des privilégiés et des appartements de ceux que le régime jugeait digne de communiquer à distance avec leur monde. Un monde dont l'orientation a été de plus en plus entravée par la disparition progressive des annuaires. Il ne néglige pas la source préférée des archéologues : les déchets.

Schlögel s'intéresse aussi à un autre butin favori des archéologues, le corps humain, et à tout ce qu'il dit sur la civilisation qui les façonne moyennant l'éducation physique du simple citoyen et du sportif jusqu'à la grâce du danseur de ballet. Il n'oublie pas de lire la présentation du corps, des habits qui le rendent identifiable comme phénomène social, des parfums qui le rendent présentable et des tatouages qui en disent énormément sur les subcultures où ils sont courants. Les pages consacrées aux industries et aux codes de la mode, au défilé historique des modèles de Christian Dior à Moscou et à la production russe et soviétique de parfums réservent de précieux enseignements sur une société où rien n'est moins simple que de trouver une paire de chaussettes décentes. Il en va de même de l'analyse subtile du régime alimentaire qui nourrit le corps - les uns mieux, les autres à peine -, de sa mission civilisatrice et de la manière dont les denrées, les plats et leurs fonctions pédagogiques apparaissent dans les livres de recette. 
La curiosité de Schlögel ne s'arrête pas aux objets plus ou moins aisément manipulables. Il s'attelle également au décryptage des messages relayés par des objets gigantesques comme le barrage monumental sur le Dniepr, l'immense ensemble d'installations métallurgiques à Magnitogorsk ou le réseau pharaonique de canaux qui relient la mer Blanche et la mer Baltique à un réseau de voies navigables qui rejoignent la mer Caspienne et la mer Noire. L'esthétisation du travail des prisonniers - qu'on nomme prudemment « guerriers du canal »-par les photos d'Aleksandr Rodčenko, l'éloge de ses vertus éducatives par une pléiade d'hommes de lettres et l'héroïsation des constructeurs de la plus grande usine sidérurgique au monde par la propagande aussi bien que par l'autoperception des ouvriers eux-mêmes fournissent des ingrédients constitutifs de l'échafaudage discursif de ce qu'on peut appeler le soviétique. La fin de l'âge du métal, l'abandon d'une bonne partie des hauts fourneaux et des fonderies et la catastrophe écologique qu'ils ont produite symbolisent à merveille l'aboutissement du projet soviétique.

Schlögel prête une attention particulière aux lieux où le projet se déploie ainsi qu'aux espaces qu'il crée. Ensemble, ils constituent le phénomène soviétique. Certains espaces sont contigus, d'autres bien éloignés les uns des autres et d'autres encore se recoupent. Il serait impossible de le décrire in extenso ou en d'en dresser un inventaire ne serait-ce qu'approximatif. Malgré tout, Schlögel réussit à offrir un panorama grâce à des exemples judicieusement choisis ainsi que par des rappels réguliers de l'environnement des objets et des situations qu'il passe en revue. Les lieux du soviétique vont des vastes régions des sites de construction ou de celles colonisées par le système des camps de concentration à la topographie révolutionnaire de Petrograd jusqu'à l'intérieur des baraques misérables qui abritent tantôt les héros de la production, tantôt les prisonniers, qui sont parfois les mêmes.

Il n'y a pas que la sphère du travail plus ou moins libre, celle des événements historiques ou celle de la vie peu ou prou privée. Il y a celle des loisirs avec les parcs de la culture et leur offre d'amusements innocents et d'activités didactiques, celle des datchas qui reflètent des hiérarchies sociales et celle des maisons de repos dont rien que l'architecture laisse entrevoir des couches archéologiques riches en enseignements. Il y a aussi l'immensité du pays où il y a mille possibilités de fuir le quotidien pour un court moment en passant des vacances. Les uns sont à la recherche de l'aventure des grandes randonnées, les autres cherchent des partenaires passagers ou stables, d'autres encore partent à la découverte de l'étranger qu'ils trouvent, faute de pouvoir quitter le pays, dans les contrées orientales de l'empire et surtout dans celles annexées pendant la guerre, où l'on peut se promener dans un décor qui rappelle le paradis interdit de l'Occident.

Les espaces les plus intéressants sont peut-être ceux qui sont bien délimités. Ils peuvent devenir des champs de bataille comme les chambres où s'affrontent les attributs du vieux monde et ceux de l'époque postrévolutionnaire, ils peuvent être des foyers d'ouvriers, d'étudiants et d'autres gens qui doivent se loger dans des fourmilières qui deviennent des melting-pots à la soviétique ou des cages d'escalier, de véritables no man's lands qui n'appartiennent à personne et du coup ne sont pas entretenus. Un espace particulier où l'homme soviétique est tempéré est 
l'appartement communautaire, la kommunalka, avec sa population hétérogène, ses rapports sociaux troubles, leurs statuts fixés et implicites qui règlent l'usage des lieux d'utilisation commune du corridor jusqu'aux toilettes et leur tendance à abolir la séparation entre l'intimité protectrice et la vie sous les yeux inquisiteurs d'un collectif condamné à cohabiter. Après la fin des années 1950, quand un programme ambitieux de construction de logements libère des millions de Soviétiques de la communauté forcée des baraques, des foyers et des kommunalki, c'est la cuisine des appartements exigus qui devient non seulement un lieu de vie privilégié, mais aussi une agora pour nombre de citoyens qui peuvent enfin discuter à haute voix des sujets qu'autrefois, ils étaient bien avisés de taire ou d'évoquer en chuchotant.

Beaucoup de chapitres évoquent des situations ainsi que les réactions et les modes de comportement qu'elles conditionnent. Les grands espaces et surtout les espaces intérieurs comme la kommunalka sont typiques à cet égard. Le calendrier révolutionnaire éphémère avec ses contraintes et avec ses fêtes ou la chorégraphie du pouvoir représentée par les défilés et par les parades livrent d'autres exemples. Les heures d'ouverture imprévisibles et le dédale des corridors des administrations ou les queues interminables devant les magasins et devant les portes d'une multitude d'organismes deviennent virtuellement institutionnalisés et standardisent des attitudes que les citoyens finissent par accepter comme des normes.

Il est impossible de faire un tour ne serait-ce qu'approximativement complet des sujets saisis, analysés et mis dans une perspective multidimensionnelle par Schlögel, qui reconnaît pour sa part que sa fresque est loin d'être achevée. Certes, elle ne pourrait l'être en aucun cas. Schlögel compose une mosaïque lacunaire qui dessine les contours d'une totalité au sens où Friedrich Hölderlin ou Georg Lukács utilisent le terme : une œuvre qui réussit à refléter un univers malgré les nombreuses composantes qui y manquent. Il révèle le message de phénomènes éparpillés du quotidien comme Siegfried Kracauer l'a fait en se promenant dans les rues de Berlin et il flâne à l'instar du Parisien Walter Benjamin (dont il se réclame) dans les labyrinthes du Soviétique en empruntant des passages qu'on découvre facilement et d'autres qui ne se révèlent que si on les cherche assidûment.

L'ouvrage n'est pas qu'une délectation pour le lecteur qui s'intéresse au phénomène soviétique. C'est une grande leçon de méthode, mais une leçon de modestie aussi pour l'historien, lequel ne devrait jamais souffrir de les recevoir. Schlögel mentionne souvent que tel ou tel autre thème mériterait des recherches plus approfondies, qu'ils sont, tels qu'il les décrit, les ébauches des projets de futurs travaux. On ne peut qu'espérer que la confrérie suive ses recommandations.

Gábor T. Rittersporn

CERCEC, CNRS, EHESS, PSL 